macrophage activation syndrome. In animals, high levels of interleukin- 6 contribute to the triggering of this syndrome ${ }^{1}$; additional cytokines, including interferon- $\gamma$, interleukin- 1 , and interleukin-18, might be involved in maintaining and amplifying the inflammatory response. ${ }^{2}$ Indeed, interleukin-1 antagonism may be beneficial in a full-blown case of the macrophage activation syndrome. ${ }^{3}$ Regarding the possible role of tocilizumab in masking the symptoms of this syndrome, the five patients with the macrophage activation syndrome reported by Shimizu et al. ${ }^{4}$ had mild laboratory abnormalities representing either blunted cases of the macrophage activation syndrome, possibly consistent with the abovementioned role of interleukin- 6 in the triggering of the syndrome, or part of the known side effects of tocilizumab (i.e., increased aminotransferase levels and neutropenia). Long-term vigilance on the rate and features of the macrophage activation syndrome during tocilizumab treatment will provide additional useful information for clinical practice.
Fabrizio De Benedetti, M.D., Ph.D.

Ospedale Pediatrico Bambino Gesù

Rome, Italy

fabrizio.debenedetti@opbg.net

Since publication of his article, the author reports no further potential conflict of interest.

1. Strippoli R, Carvello F, Scianaro R, et al. Amplification of the response to toll-like receptor ligands by prolonged exposure to interleukin- 6 in mice: implication for the pathogenesis of macrophage activation syndrome. Arthritis Rheum 2012;64: 1680-8.

2. Strippoli R, Caiello I, De Benedetti F. Reaching the threshold: a multilayer pathogenesis of macrophage activation syndrome. J Rheumatol (in press).

3. Miettunen PM, Narendran A, Jayanthan A, Behrens EM, Cron RQ. Successful treatment of severe paediatric rheumatic disease-associated macrophage activation syndrome with interleukin-1 inhibition following conventional immunosuppressive therapy: case series with 12 patients. Rheumatology (Oxford) 2011;50:417-9.

4. Shimizu M, Nakagishi Y, Kasai K, et al. Tocilizumab masks the clinical symptoms of systemic juvenile idiopathic arthritisassociated macrophage activation syndrome: the diagnostic significance of interleukin-18 and interleukin-6. Cytokine 2012;58: 287-94.

DOI: 10.1056/NEJMc1301017

\title{
Tolvaptan in Autosomal Dominant Polycystic Kidney Disease
}

TO THE EDITOR: In the Tolvaptan Efficacy and Safety in Management of Autosomal Dominant Polycystic Kidney Disease and Its Outcome (TEMPO) 3:4 trial, Torres et al. (Dec. 20 issue) ${ }^{1}$ found that tolvaptan slowed the increase in renal volume and the decline in renal function, as compared with placebo, in patients with autosomal dominant polycystic kidney disease (ADPKD). Given that the beneficial effect of tolvaptan is thought to operate through the inhibition of $\mathrm{V}_{2}$-receptor activation and the suppression of cyclic AMP (cAMP), it seems likely that a similarly beneficial effect on the course of ADPKD could be achieved with a high fluid intake alone, because this suppresses vasopressin release and cAMP formation. ${ }^{2}$ Therefore, it is hard to understand why the investigators did not instruct both groups to ingest large amounts of water, as two of the authors had previously recommended. ${ }^{2}$ Had they done so, we would have known whether tolvaptan is superior to a high fluid intake alone. In view of the worrisome adverse effects of tolvap$\tan$ seen in the trial, including elevated liverenzyme levels, as well as the extremely high cost of tolvaptan (cost of daily 90 -mg dose $>\$ 25,000$ per month ${ }^{3}$ ), a monitored high water intake may be safer, far cheaper, and equally effective.

Aaron Spital, M.D.

St. Luke's Hospital

New York, NY

aspital@att.net

No potential conflict of interest relevant to this letter was reported.

1. Torres VE, Chapman AB, Devuyst $\mathrm{O}$, et al. Tolvaptan in patients with autosomal dominant polycystic kidney disease. $\mathrm{N}$ Engl J Med 2012;367:2407-18.

2. Torres VE, Bankir L, Grantham JJ. A case for water in the treatment of polycystic kidney disease. Clin J Am Soc Nephrol 2009;4:1140-50.

3. HealthTrans. Pharmacy prices (http://cashcard.lc.healthtrans .com/Pages/DrugPrices.aspx).

DOI: 10.1056/NEJMc1300762

TO THE EDITOR: Torres et al. found that tolvaptan slowed the decline in kidney function in patients with ADPKD. One small, short-term study ${ }^{1}$ showed a reversible decline in the glomerular filtration rate (GFR) and an increase of approximately $8 \%$ in the serum creatinine after the initiation of tolvaptan in patients with ADPKD. 
Therefore, in the study by Torres et al., the kidneyfunction end points were assessed after the doseescalation phase was completed.

Did this larger study find a similar effect? Before there is widespread use of tolvaptan in patients with ADPKD, it would be important for practitioners to be aware of the expected average and range of the increase in the creatinine level after the initiation of tolvaptan and whether this would be reversible after 3 years of therapy. Is there a percentage increase in creatinine at which tolvaptan therapy should be stopped?

It is interesting to note that this effect has not been reported when tolvaptan has been used in large studies of hyponatremia or heart failure, although at lower doses than were used in the study by Torres et al. This raises questions about whether this is a dose-related effect or whether it is specific to ADPKD.

Ajay Kher, M.B., B.S.

University of Massachusetts Memorial Medical Center Worcester, MA

ajay.kher@umassmemorial.org

No potential conflict of interest relevant to this letter was reported.

1. Irazabal MV, Torres VE, Hogan MC, et al. Short-term effects of tolvaptan on renal function and volume in patients with autosomal dominant polycystic kidney disease. Kidney Int 2011;80: 295-301.

DOI: 10.1056/NEJMc1300762

TO THE EDITOR: In a prespecified subgroup analysis, tolvaptan had a beneficial effect on total kidney volume in all subgroups specified according to baseline values for total kidney volume $(<1500 \mathrm{ml}$ vs. $\geq 1500 \mathrm{ml}$ ).

Ho et al. examined the central and nephrogenic components of osmoregulation with the use of water-deprivation tests in 10 adults and 10 children with ADPKD and normal renal function. ${ }^{1}$ These patients had a peripheral resistance to vasopressin, the extent of which was directly correlated with total kidney volume, as assessed on magnetic resonance imaging.

One might hypothesize that if the nephrogenic resistance to vasopressin is related to total kidney volume, then perhaps the beneficial effects of vasopressin antagonism will be greater in patients with a lower total kidney volume at the start of treatment, as compared with those with a higher total kidney volume, since patients with a lower volume may be inherently more sensitive to the deleterious effects of vasopres- sin. Was the slowing of the increase in total kidney volume associated with a baseline value that was defined as a continuous variable, rather than dichotomized into groups $(<1500 \mathrm{ml}$ vs. $\geq 1500 \mathrm{ml}$ )? One might expect that patients with a lower total kidney volume at baseline had a greater reduction in the rate of growth in total kidney volume, as compared with those with a higher total kidney volume at baseline.

Donal J. Sexton, M.D.

National University of Ireland Galway

Galway, Ireland

donalsexton0@hotmail.com

No potential conflict of interest relevant to this letter was reported.

1. Ho TA, Godefroid N, Gruzon D, et al. Autosomal dominant polycystic kidney disease is associated with central and nephrogenic defects in osmoregulation. Kidney Int 2012;82:1121-9.

DOI: $10.1056 / N E J M c 1300762$

TO THE EDITOR: A history of urinary tract infection is regarded as an independent prognostic factor for a decline in GFR in patients with ADPKD. ${ }^{1}$ Furthermore, evidence indicates substantial overlap between biologic processes involved in cystogenesis and in kidney injury, with macrophages having a key role. ${ }^{2,3}$ In the TEMPO 3:4 trial, patients who received tolvaptan had a significantly lower rate of urinary tract infection and renal cyst infection, as compared with patients who received placebo. ${ }^{4}$ This finding probably reflects the importance of polyuria and pollakiuria in preventing urinary tract infection. ${ }^{5}$ On the basis of the emerging concept of renal inflammation as an essential pathogenic component of naturally progressing cystic kidney disease, we speculate that the lower incidence of urinary tract infection and renal cyst infection observed in the tolvaptan group contributes to the potential benefit of this drug on the decline in GFR in patients with ADPKD. Further prospective trials comparing the use and nonuse of tolvaptan in patients with ADPKD with equivalent urinary volume and frequency would provide data regarding the protective effect of tolvaptan per se.

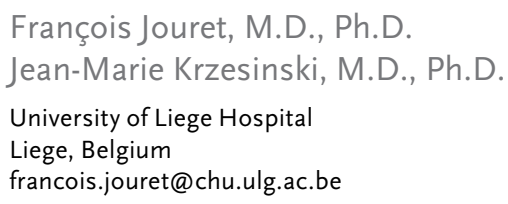

No potential conflict of interest relevant to this letter was reported. 
1. Rule $\mathrm{AD}$, Torres $\mathrm{VE}$, Chapman $\mathrm{AB}$, et al. Comparison of methods for determining renal function decline in early autosomal dominant polycystic kidney disease: the consortium of radiologic imaging studies of polycystic kidney disease cohort. J Am Soc Nephrol 2006;17:854-62.

2. Huang da W, Sherman BT, Lempicki RA. Systematic and integrative analysis of large gene lists using DAVID bioinformatics resources. Nat Protoc 2009;4:44-57.

3. Karihaloo A, Koraishy F, Huen SC, et al. Macrophages promote cyst growth in polycystic kidney disease. J Am Soc Nephrol 2011;22:1809-14

4. Torres VE, Chapman AB, Devuyst $\mathrm{O}$, et al. Tolvaptan in patients with autosomal dominant polycystic kidney disease. $\mathrm{N}$ Engl J Med 2012;367:2407-18.

5. Hooton TM. Uncomplicated urinary tract infection. $\mathrm{N}$ Engl J Med 2012;366:1028-37.

DOI: 10.1056/NEJMc1300762

THE AUTHORS REPLY: Spital points out that increasing water intake may slow down the progression of ADPKD. ${ }^{1}$ However, adherence to a regimen of high water intake that would be sufficient to suppress vasopressin during prolonged periods of time may be difficult ${ }^{2}$ and, as some authors have suggested, possibly deleterious. ${ }^{3}$ In the TEMPO 3:4 study, all patients were encouraged to drink enough water to avoid dehydration. As a result, water intake in placebo-treated patients increased, as reflected by the reduction of median nonfasting first-morning-urine osmolality from a baseline value of 511 mOsm per kilogram of water (95\% confidence interval [CI], 164 to 863 ) to 438 mOsm per kilogram (95\% CI, 159 to 792) during the trial. A specifically designed clinical trial would be necessary to determine whether high water intake and tolvaptan are equally effective treatments. Tolvaptan has not been approved for the treatment of ADPKD; therefore, pricing information is not available.

Kher raises important questions regarding creatinine levels. The median serum creatinine level in the tolvaptan group increased from $1.00 \mathrm{mg}$ per deciliter (95\% CI, 0.61 to 1.76 ) to $1.06 \mathrm{mg}$ per deciliter (95\% CI, 0.64 to 1.88 ; median change, $0.05 \mathrm{mg}$ per deciliter [ $95 \% \mathrm{CI},-0.14$ to 0.30 ]) during the dose-escalation phase and decreased from $1.14 \mathrm{mg}$ per deciliter (95\% CI, 0.67 to 2.47 ) to $1.08 \mathrm{mg}$ per deciliter ( $95 \% \mathrm{CI}, 0.63$ to 2.33; median change, $-0.04 \mathrm{mg}$ per deciliter [95\% CI, -0.32 to 0.16$]$ ) during the washout phase. The initial, reversible reduction in GFR probably reflects the reversal of the dampening effect of vasopressin on tubuloglomerular feedback. ${ }^{4}$ As such, it is expected to be dose-related and not specific to ADPKD. It is premature to speculate on the increase in serum creatinine that would require the discontinuation of tolvaptan, since the drug is not currently approved for the treatment of ADPKD.

Sexton suggests that patients with a total kidney volume of $1500 \mathrm{ml}$ or more may benefit the least from tolvaptan, since in such patients vasopressin is less likely to result in a maximum urine concentration. This hypothesis would be reasonable if the concentrating defect was due to reduced production or increased degradation of cAMP or decreased protein kinase A activity, since tolvaptan is thought to inhibit cyst growth by restraining cAMP and protein kinase A signaling. However, the concentrating defect in patients with ADPKD is probably due to downstream cellular processes or to the reduced efficiency of countercurrent exchange caused by disruption of the medullary architecture. ${ }^{5}$ This is consistent with the similar reductions (approximately 50\%) in the rate of growth in total kidney volume that was observed in the two groups of patients (total kidney volume, $<1500 \mathrm{ml}$ or $\geq 1500 \mathrm{ml}$ ), as shown in Figure $2 \mathrm{~B}$ of the article.

We concur with the comments of Jouret and Krzesinski regarding urinary tract infection and renal cyst infection in patients with ADPKD.

Vicente E. Torres, M.D., Ph.D.

Mayo Clinic

Rochester, MN

torres.vicente@mayo.edu

Ron T. Gansevoort, M.D., Ph.D.

University of Groningen

Groningen, the Netherlands

Frank S. Czerwiec, M.D., Ph.D.

Otsuka Pharmaceutical Development and Commercialization Rockville, MD

Since publication of their article, the authors report no further potential conflict of interest.

1. Torres VE, Bankir L, Grantham JJ. A case for water in the treatment of polycystic kidney disease. Clin J Am Soc Nephrol 2009;4:1140-50.

2. Magpantay L, Ziai F, Oberbauer R, Haas M. The effect of fluid intake on chronic kidney transplant failure: a pilot study. J Ren Nutr 2011;21:499-505.

3. Hebert LA, Greene T, Levey A, Falkenhain ME, Klahr S. High urine volume and low urine osmolality are risk factors for faster progression of renal disease. Am J Kidney Dis 2003;41:962-71.

4. Bouby N, Ahloulay M, Nsegbe E, Déchaux M, Schmitt F, Bankir L. Vasopressin increases glomerular filtration rate in conscious rats through its antidiuretic action. J Am Soc Nephrol 1996;7:842-51.

5. Bankir L, Bichet DG. Polycystic kidney disease: an early urea-selective urine-concentrating defect in ADPKD. Nat Rev Nephrol 2012;8:437-9.

DOI: $10.1056 / N E J M c 1300762$ 